\title{
Veterinary Drugs Found in Pharmacies in Turkey
}

\section{Dilsad ONBASLI*, Gokcen YUVALI CELIK, Ahmet CEYLAN, Merve YUCETURK Azranur DAL}

Erciyes Üniversitesi, Eczacılık Fakültesi, Farmasötik Biyoteknoloji Anabilim Dalı, 38039, Kayseri-Türkiye

*Correspondence:

Dilsad ONBASLI

Erciyes Üniversitesi, Eczacılık Fakültesi, Farmasötik Biyoteknoloji Anabilim Dalı, 38039, Kayseri-Türkiye

\begin{abstract}
In this study veterinary drugs in RxMediapharma 2017 which actively used in pharmacies in Turkey were scanned. These veterinary drugs offered for sale in pharmacies are given active substances, animals used, drug form, route of application, local or imported. According to this result, Ministry of Health Licensed 1; Ministry of Food, Agriculture and Livestock Licensed 56 local and 23 imported and a total of 79 veterinary drugs were detected. In addition, when the Ministry of Food, Agriculture and Livestock Licensed drugs are grouped according to their pharmacological effects, it is determined that the licensed drug group which has the highest number with $43.75 \%$ is antibiotics. Although there are various studies concerning veterinary drug, İt is given as Turkey-scale all of these drugs has not been observed in an article expect e-data, and the data collected electronically, has been submitted to any scientific community. For this reason, it is thought that this study, which compiles veterinary drugs emphasizing therapeutic potential, will form the basis for future research.
\end{abstract}

Keywords: Veterinary drugs, pharmacy, Turkey, Ministry of health, Ministry of food- agriculture and livestock, RxMediapharma,

\section{Introduction}

Veterinary drugs; is a combination of chemicals and chemical substances used to preserve and improve animal health (Yarsan 2015). Since ancient times, veterinary medicines have been used to treat diseases, to prevent diseases, to increase efficiency, to accelerate the development and growth of animals (Ozturk 2002). Veterinary medicine in which these substances are used has a large number of preparations belonging to various pharmacological groups such as vitamins, mineral supplements, antibiotics, analgesics and electrolytes as well as human medicine (DPT 2001).

In veterinary medicine; the use of medicines for different purposes in the treatment of animal diseases. Therefore, veterinarians; they use the electronic environment in which detailed information about the medicines they use is contained. Although the studies about this issue with some veterinary drugs, veterinary medicines including a drug database through Turkey does not have a working scale. In this study, a drug database that RxMediapharma ${ }^{\circledR}$ 2017, based on the Ministry of Health found in pharmacies in Turkey licensed and Ministry of Food, Agriculture and Livestock subtracting list of licensed veterinary pharmaceutical active ingredients in the content of these drugs, animals are used, drug form, route of administration, local or import status and the location of the treatment.

\section{Methods}

In this study, based on RxMediapharma ${ }^{\circledR} 2017$, is located in pharmacies in Turkey in 2017, the Ministry of Health and Ministry of Food, Agriculture and Livestock licensed local and imported veterinary drugs were examined.

\section{Results}


In this study, according to RxMediapharma ${ }^{\circledR} 2017$ data, vitamins, mineral supplements, electrolytes, antibiotics (systemic, local), anthelmintics, ectoparasites, antiprotozoals, endectosides, antiinflamatuaries, analgesics, antirheumatic drugs, gastrointestinal system drugs, anticholinergics drugs, analeptic drugs, genitourinary system drugs and sex hormones, hormones, antiseptics and disinfectants, non-therapeutic products are screened and the preparations, the active substances of the preparations, the animals used, the drug form, the route of administration, local or imported status are given in Table 1-17 respectively.

Table 1: Ministry of Food, Agriculture and Livestock (MFAL) Licensed Vitamins

\begin{tabular}{|c|c|c|c|c|}
\hline Drugs & Active substances & $\begin{array}{l}\text { Animal used of } \\
\text { the drugs }\end{array}$ & $\begin{array}{l}\text { Routes of } \\
\text { Administration }\end{array}$ & Local/ Imported \\
\hline Adevet & $\begin{array}{l}\text { Vitamin } \mathrm{D}_{3}(75.000 \\
\mathrm{IU}) \\
\text { Vitamin E (50 IU) } \\
\text { Retinol Propionate } \\
\text { (500.000 IU) }\end{array}$ & $\begin{array}{l}\text { Horse, Goat, } \\
\text { Sheep, Lamb, } \\
\text { Rabbit, } \\
\text { Capricorn, Cattle }\end{array}$ & $\begin{array}{l}\text { IM (Intramuscular), } \\
\text { SC(Subcutaneous ) }\end{array}$ & Local \\
\hline $\begin{array}{l}\text { Ademin } \\
\text { Injectable } \\
\text { Solution }\end{array}$ & $\begin{array}{l}\text { Vitamin A }(500.000 \\
\text { IU) } \\
\text { Vitamin E }(50 \mathrm{mg}) \\
\text { Vitamin } \mathrm{D}_{3} \quad(75.000 \\
\text { IU) }\end{array}$ & $\begin{array}{lr}\text { Veal, } & \text { Moss, } \\
\text { Heifer, } & \text { Goat, } \\
\text { Sheep, } & \text { Lamb, } \\
\text { Rabbit, Cattle }\end{array}$ & $\mathrm{IM}, \mathrm{SC}$ & Local \\
\hline $\begin{array}{l}\text { Akavit } \\
\text { Oral } \\
\text { Powder for } \\
\text { Solution }\end{array}$ & $\begin{array}{l}\text { Vitamin } \mathrm{K}_{3}(10 \mathrm{mg}) \\
\text { Retinol Acetate } \\
(25.000 \mathrm{IU})\end{array}$ & $\begin{array}{l}\text { Calf, } \quad \text { Chick, } \\
\text { Turkey, Goat, } \\
\text { Sheep, Lamb, } \\
\text { Capricorn, } \\
\text { Chicken, Cattle }\end{array}$ & Oral & Local \\
\hline $\begin{array}{l}\text { Bakosel } \\
\text { Soft } \\
\text { Gelatin } \\
\text { Capsule }\end{array}$ & $\begin{array}{l}\text { Dicalcium phosphate } \\
(150 \mathrm{mg}) \\
\text { Vitamin E (500 IU) } \\
\text { Sodium selenite ( } 2.5 \\
\text { mg) } \\
\text { Copper sulphate ( } 10 \\
\text { mg) } \\
\text { Cobalt (II) sülfat12.5 } \\
\text { mg) }\end{array}$ & $\begin{array}{l}\text { Calf, } \\
\text { Heifer, } \\
\text { Goat, } \\
\text { Lamb, } \\
\text { Lattle }\end{array}$ & Oral & Local \\
\hline $\begin{array}{l}\text { Berovit } B_{12} \\
\text { Injectable } \\
\text { Solution }\end{array}$ & $\begin{array}{l}\text { D-Pantenol }(10 \mathrm{mg}) \\
\text { Niasinamit }(20 \mathrm{mg}) \\
\text { Vitamin } B_{12}(4 \mathrm{mg}) \\
\text { Vitamin } B_{1}(5 \mathrm{mg}) \\
\text { Vitamin } B_{6}(2 \mathrm{mg}) \\
\text { Vitamin } B_{2}(2 \mathrm{mg})\end{array}$ & $\begin{array}{lr}\text { Calf, } & \text { Beef, } \\
\text { Heifer, } & \text { Moss, } \\
\text { Goat, } & \text { Sheep, } \\
\text { Lamb, Capricorn, } \\
\text { Cattle, Cat, Dog, } \\
\begin{array}{l}\text { Mandate, } \\
\text { Horse }\end{array}\end{array}$ & $\mathrm{IM}, \mathrm{SC}$ & Local \\
\hline $\begin{array}{l}\text { Injacom-C } \\
\text { Injectable } \\
\text { Solution }\end{array}$ & Vitamin C (200 mg) & $\begin{array}{l}\text { Horse, Calf, Veal, } \\
\text { Heifer, Moss, } \\
\text { Goat, Sheep, } \\
\text { Cattle, Cat, Dog, }\end{array}$ & $\begin{array}{l}\text { IV( Intravenous ), } \\
\text { IM }\end{array}$ & Local \\
\hline $\begin{array}{l}\text { Thiabral } \\
\text { Injectable } \\
\text { Solution }\end{array}$ & $\begin{array}{l}\text { Vitamin } B_{1}(5.75 \mathrm{~g}) \\
\text { Vitamin } B_{6}(0.55 \mathrm{~g})\end{array}$ & $\begin{array}{l}\text { Horse, Calf, Goat, } \\
\text { Sheep, Cat, Dog, } \\
\text { Cattle, Thai }\end{array}$ & IV, IM & Local \\
\hline $\begin{array}{l}\text { Yeldif } \\
\text { Injectable } \\
\text { Suspension }\end{array}$ & $\begin{array}{l}\text { Vitamin } B_{1}(40 \mathrm{mg}) \\
\text { Vitamin E }(60 \mathrm{mg}) \\
\text { Sodium selenite } \\
(1 \mathrm{mg})\end{array}$ & $\begin{array}{l}\text { Horse, Calf, Goat, } \\
\text { Sheep (Pregnant), } \\
\text { Lamb, Cattle } \\
\text { (Pregnant) }\end{array}$ & $\mathrm{IM}, \mathrm{SC}$ & Local \\
\hline
\end{tabular}

Table 2: MFAL Licensed Mineral Suplements

\begin{tabular}{|l|l|l|l|l|}
\hline Drugs & Active substances & $\begin{array}{l}\text { Animal used of } \\
\text { the drugs }\end{array}$ & $\begin{array}{l}\text { Routes of } \\
\text { Administration }\end{array}$ & Local/ Imported \\
\hline Kaldif & Boronic Acid (7.29 & Goat, Sheep, & IV, SC & Local \\
\hline
\end{tabular}




\begin{tabular}{|c|c|c|c|c|}
\hline $\begin{array}{l}\text { Fort } \\
\text { Injectable } \\
\text { Solution }\end{array}$ & $\begin{array}{l}\text { g) } \\
\text { Calcium Gluconate } \\
(38.71 \mathrm{~g}) \\
\text { Magnesium } \\
\text { Chloride }(6.5 \mathrm{~g}) \\
\text { Calcium Hydroxide } \\
(1.32 \mathrm{~g})\end{array}$ & Cattle & & \\
\hline $\begin{array}{l}\text { Tildren } \\
\text { Injectable } \\
\text { Powder } \\
\text { for } \\
\text { Solution }\end{array}$ & $\begin{array}{l}\text { Tilundronic Acid } \\
(50 \mathrm{mg})\end{array}$ & Horse & Perfusion, IV & Imported \\
\hline
\end{tabular}

Table 3: MFAL Licensed Electrolytes

\begin{tabular}{|c|c|c|c|c|}
\hline Drugs & Active substances & $\begin{array}{l}\text { Animal } \\
\text { used of the } \\
\text { drugs }\end{array}$ & $\begin{array}{ll}\text { Routes } & \text { of } \\
\text { Administration } & \end{array}$ & Local / Imported \\
\hline $\begin{array}{l}\text { Glutellac } \\
\text { Oral Solution }\end{array}$ & $\begin{array}{l}\text { Dextrose } \\
\text { monohydrate }(19.5 \\
\mathrm{g}) \\
\text { Potassium } \\
\text { chloride }(3.2 \mathrm{~g}) \\
\text { Sodium acetate } \\
(18.32 \mathrm{~g}) \\
\text { Sodium chloride } \\
(3.2 \mathrm{~g}) \\
\text { Sodium diacetate } \\
(1.6 \mathrm{~g})\end{array}$ & Calf, Cattle & Oral & Imported \\
\hline $\begin{array}{l}\text { Rehydion } \\
\text { Gel }\end{array}$ & $\begin{array}{l}\text { Potassium }(4.5 \%) \\
\text { Sodium }(11.5 \%) \\
\text { Chloride }(8.6 \%)\end{array}$ & Calf, Cattle & Oral & Imported \\
\hline
\end{tabular}

Table 4: MFAL Licensed Systemic Antibiotics

\begin{tabular}{|c|c|c|c|c|}
\hline Drugs & Active substances & $\begin{array}{l}\text { Animal } \\
\text { used of the } \\
\text { drugs }\end{array}$ & $\begin{array}{l}\text { Routes of } \\
\text { Administration }\end{array}$ & Local/ Imported \\
\hline $\begin{array}{l}\text { Animar } \\
\text { Injectable } \\
\text { Solution }\end{array}$ & $\begin{array}{l}\text { Trimetoprim } \\
\text { (40mg) } \\
\text { Sulfadoxine } \\
(200 \mathrm{mg})\end{array}$ & $\begin{array}{l}\text { Horse, } \\
\text { Beef, } \\
\text { Heifer, } \\
\text { Moss, Goat, } \\
\text { Sheep and } \\
\text { Dog }\end{array}$ & IV, IM, SC & Local \\
\hline $\begin{array}{l}\text { Biotrin Oral- } \\
\text { Intrauterine } \\
\text { Tablet }\end{array}$ & $\begin{array}{l}\text { Sulfadiazine } \\
(1000 \mathrm{mg}) \\
\text { Trimethoprim } \\
(200 \mathrm{mg})\end{array}$ & $\begin{array}{l}\text { Goat, } \\
\text { Sheep, Cat, } \\
\text { Calf, Horse }\end{array}$ & Oral & Local \\
\hline $\begin{array}{l}\text { Cenmicin- } \\
\text { LC } \\
\text { Injectable } \\
\text { Solution }\end{array}$ & $\begin{array}{l}\text { Sipektinomycin } \\
(100 \mathrm{mg}) \\
\text { Linkomycin } \\
\mathrm{mg})\end{array}$ & $\begin{array}{l}\text { Sheep, } \\
\text { Cattle }\end{array}$ & Injection and Other & Imported \\
\hline $\begin{array}{l}\text { Cevaxel Rtu } \\
\text { Injectable } \\
\text { Suspension }\end{array}$ & Ceftiofur $(50 \mathrm{mg})$ & Cattle & $\mathrm{SC}$ & Imported \\
\hline
\end{tabular}




\begin{tabular}{|c|c|c|c|c|}
\hline $\begin{array}{l}\text { Danomed } \\
\text { Oral Powder } \\
\text { for Solution }\end{array}$ & $\begin{array}{l}\text { Danofloxacin } \\
(100 \mathrm{mg})\end{array}$ & $\begin{array}{l}\text { Turkey and } \\
\text { Chicken } \\
\text { (fed for } \\
\text { meat) }\end{array}$ & Oral & Local \\
\hline $\begin{array}{l}\text { Devamisin } \\
\text { Oral / Intra- } \\
\text { uterine } \\
\text { Tablet }\end{array}$ & $\begin{array}{l}\text { Chlortetracycline } \\
(500 \mathrm{mg})\end{array}$ & $\begin{array}{l}\text { Horse, } \\
\text { Goat, } \\
\text { Sheep, } \\
\text { Cattle }\end{array}$ & Oral, Intra-uterine & Local \\
\hline $\begin{array}{l}\text { Diaqua } \\
\text { Oral } \\
\text { Powder }\end{array}$ & $\begin{array}{l}\text { Sulfadiazine } \\
(416.7 \mathrm{mg}) \\
\text { Trimetoprim } \\
(83.3 \mathrm{mg})\end{array}$ & Fish & Oral & Local \\
\hline $\begin{array}{l}\text { Epimycin } \\
\text { Powder for } \\
\text { Solution }\end{array}$ & $\begin{array}{l}\text { Erythromycin }(210 \\
\mathrm{mg})\end{array}$ & Chicken & Oral & Local \\
\hline $\begin{array}{l}\text { Ertruth Oral } \\
\text { Solution }\end{array}$ & $\begin{array}{l}\text { Erythromycin }(350 \\
\mathrm{mg})\end{array}$ & Chicken & Oral & Local \\
\hline $\begin{array}{l}\text { Florkem } \\
\text { Injectable } \\
\text { Solution }\end{array}$ & Florfenicol & Cattle & $\mathrm{IM}, \mathrm{SC}$ & Local \\
\hline $\begin{array}{l}\text { Gabbrocol } \\
\text { Powder for } \\
\text { Oral Solution }\end{array}$ & $\begin{array}{l}\text { Paramomycin }(70 \\
\mathrm{mg})\end{array}$ & Calf & Oral & Imported \\
\hline $\begin{array}{l}\text { Marbox } \\
\text { Injectable } \\
\text { Solution }\end{array}$ & Marbofloxacin & Cattle & IV, IM, SC & Imported \\
\hline $\begin{array}{l}\text { Medicalin } \\
\text { Powder for } \\
\text { Oral Solution }\end{array}$ & Lincomycin (\% 40$)$ & $\begin{array}{l}\text { Chicken, } \\
\text { Turkey }\end{array}$ & Oral & Local \\
\hline $\begin{array}{l}\text { Medicamox } \\
\text { Powder for } \\
\text { Oral Solution }\end{array}$ & $\begin{array}{l}\text { Amoxicillin } \quad(500 \\
\mathrm{mg})\end{array}$ & $\begin{array}{l}\text { Chicken, } \\
\text { Turkey (fed } \\
\text { for meat) }\end{array}$ & Oral & Local \\
\hline $\begin{array}{l}\text { Medicatey } \\
\text { Powder for } \\
\text { Oral Solution }\end{array}$ & Tylosin (\% 100) & $\begin{array}{l}\text { Chicken, } \\
\text { Turkey (fed } \\
\text { for meat) }\end{array}$ & Oral & Local \\
\hline $\begin{array}{l}\text { Mediflor } \\
\text { Oral Solution }\end{array}$ & $\begin{array}{l}\text { Florfenicol } \quad(300 \\
\mathrm{mg})\end{array}$ & $\begin{array}{l}\text { Chicken } \\
\text { (fed for } \\
\text { meat) }\end{array}$ & Oral & Local \\
\hline $\begin{array}{l}\text { Moksidif La } \\
\text { Injectable } \\
\text { Suspension }\end{array}$ & $\begin{array}{l}\text { Amoxicillin }(150 \\
\mathrm{mg})\end{array}$ & $\begin{array}{l}\text { Sheep, } \\
\text { Cattle }\end{array}$ & IM & Imported \\
\hline $\begin{array}{l}\text { Neo-500 } \\
\text { Powder for } \\
\text { Oral Solution }\end{array}$ & Neomycin(500 mg) & $\begin{array}{l}\text { Horse, } \\
\text { Turkey, } \\
\text { Chicken, } \\
\text { Calf, } \\
\text { Capricorn, } \\
\text { Sheep }\end{array}$ & Oral & Local \\
\hline $\begin{array}{l}\text { Nuflor } \\
\text { Minidose } \\
\text { Injectable } \\
\text { Solution } \\
\end{array}$ & $\begin{array}{l}\text { Florfenicol }(450 \\
\mathrm{mg})\end{array}$ & Cattle & IM, SC & Imported \\
\hline $\begin{array}{l}\text { Preventium } \\
\text { Powder for }\end{array}$ & Tiamulin(450 mg) & $\begin{array}{l}\text { Chicken, } \\
\text { Turkey (fed }\end{array}$ & Oral & Local \\
\hline
\end{tabular}




\begin{tabular}{|c|c|c|c|c|}
\hline Oral Solution & & for meat) & & \\
\hline $\begin{array}{l}\text { Proflox } \\
\text { Injectable } \\
\text { Solution }\end{array}$ & $\begin{array}{l}\text { Enrofloxacin }(130 \\
\mathrm{mg})\end{array}$ & $\begin{array}{l}\text { Sheep, } \\
\text { Cattle }\end{array}$ & IV, IM, SC & Local \\
\hline $\begin{array}{l}\text { Spectam } \\
\text { Oral Gel }\end{array}$ & $\begin{array}{l}\text { Spectinomycin }(50 \\
\mathrm{mg})\end{array}$ & Lamb, Calf & Oral & Local \\
\hline $\begin{array}{l}\text { Sulphamezat } \\
\text { hine \% } 16 \\
\text { Oral Solution }\end{array}$ & $\begin{array}{l}\text { Sulfadimidine (160 } \\
\mathrm{mg})\end{array}$ & $\begin{array}{l}\text { Turkey (fed } \\
\text { for meat), } \\
\text { Goat, } \\
\text { Sheep, } \\
\text { Cattle, } \\
\text { Chicken } \\
\text { (fed for } \\
\text { meat) }\end{array}$ & Oral & Local \\
\hline $\begin{array}{l}\text { Spirovet } \\
\text { Injectable } \\
\text { Solution }\end{array}$ & $\begin{array}{l}\text { Spiramycin } \\
(600.000 \mathrm{IU})\end{array}$ & Calf, Cattle & IM & Imported \\
\hline $\begin{array}{l}\text { Tenaline La } \\
\text { Injectable } \\
\text { Solution } \\
\end{array}$ & $\begin{array}{l}\text { Oxytetracycline } \\
(200 \mathrm{mg})\end{array}$ & $\begin{array}{l}\text { Goat, } \\
\text { Cattle, } \\
\text { Sheep }\end{array}$ & IM & Imported \\
\hline $\begin{array}{l}\text { Tetradil } \\
\text { Powder } \\
\text { for Oral } \\
\text { Solution }\end{array}$ & $\begin{array}{l}\text { Oxytetracycline } \\
(120 \mathrm{mg}) \\
\text { Sulfadimidine } \\
(800 \mathrm{mg}))\end{array}$ & $\begin{array}{l}\text { Dog, } \\
\text { Calf, } \\
\text { Lamb, } \\
\text { Thai }\end{array}$ & Oral & Local \\
\hline $\begin{array}{l}\text { Tetramed } \\
\text { Powder for } \\
\text { Oral Solution } \\
\end{array}$ & $\begin{array}{l}\text { Tetracycline } \quad(500 \\
\mathrm{mg})\end{array}$ & $\begin{array}{l}\text { Turkey, } \\
\text { Chicken, } \\
\text { Calf }\end{array}$ & Oral & Local \\
\hline $\begin{array}{l}\text { Tetramezatin } \\
\text { Powder for } \\
\text { Oral Solution }\end{array}$ & $\begin{array}{l}\text { Oxytetracycline } \\
(0.3 \mathrm{~g}) \\
\text { Sulfadimidine }(2 \mathrm{~g})\end{array}$ & $\begin{array}{l}\text { Capricorn, } \\
\text { Calf, Dog, } \\
\text { Lamb, Thai }\end{array}$ & Oral & Local \\
\hline $\begin{array}{l}\text { Tetramisin } \\
\text { Injectable } \\
\text { Solution } \\
\end{array}$ & $\begin{array}{l}\text { Oxytetracycline } \\
(30 \mathrm{mg}) \\
\text { Lidocaine }(20 \mathrm{mg})\end{array}$ & $\begin{array}{l}\text { Horse, Cat, } \\
\text { Dog }\end{array}$ & IV, IM, SC & Local \\
\hline Tiamvet 100 & Tiamulin $(10 \mathrm{~g})$ & Chicken & Oral & Imported \\
\hline $\begin{array}{l}\text { Tilocen-20 } \\
\text { Injectable } \\
\text { Solution }\end{array}$ & Tylosin $(200 \mathrm{mg})$ & $\begin{array}{l}\text { Cattle, } \\
\text { Sheep }\end{array}$ & IM & Imported \\
\hline $\begin{array}{l}\text { Vetakoksin } \\
\text { Oral Solution }\end{array}$ & $\begin{array}{l}\text { Sulfaquinoxaline } \\
(32 \mathrm{mg})\end{array}$ & $\begin{array}{l}\text { Pigeon, } \\
\text { Chick, } \\
\text { Budgerigar, } \\
\text { Parrot, } \\
\text { Canary, } \\
\text { Pheasant, } \\
\text { Chicken } \\
\text { (fed for } \\
\text { meat), } \\
\text { Turkey (fed } \\
\text { for meat) }\end{array}$ & Oral & Local \\
\hline
\end{tabular}

Table 5: MFAL Licensed Local Antibiotics

\begin{tabular}{|c|c|c|c|c|}
\hline Drugs & $\begin{array}{l}\text { Active } \\
\text { substances }\end{array}$ & $\begin{array}{l}\text { Animal used } \\
\text { of the drugs }\end{array}$ & $\begin{array}{l}\text { Routes of } \\
\text { Administration }\end{array}$ & $\begin{array}{l}\text { Local/ } \\
\text { Imported }\end{array}$ \\
\hline Gentavet-D & Gentamicin & Rabbit,Turkey, & Topical & Local \\
\hline
\end{tabular}




\begin{tabular}{|l|l|l|l|l|}
\hline $\begin{array}{l}\text { Skin } \\
\text { Ointment }\end{array}$ & $\begin{array}{l}\text { Horse, Dog, } \\
\text { Sheep, Goat, } \\
\text { Cattle }\end{array}$ & & \\
\hline $\begin{array}{l}\text { Lincogemast } \\
\text { In-breast } \\
\text { ointment }\end{array}$ & $\begin{array}{l}\text { Gentamicin } \\
(80,000 \mathrm{IU}) \\
\text { Linkomycin } \\
(250,000 \mathrm{IU}) \\
\text { Prednisolone } \\
(5 \mathrm{mg})\end{array}$ & Cattle & In-breast & Imported \\
\hline $\begin{array}{l}\text { Piyedif NG } \\
\text { Skin Spray }\end{array}$ & $\begin{array}{l}\text { Thiamphenicol } \\
(2.85 \%)\end{array}$ & All species & Topical & Imported \\
\hline
\end{tabular}

Table 6: MFAL Licensed Antihelmintics

\begin{tabular}{|c|c|c|c|c|}
\hline Drugs & Active substances & $\begin{array}{l}\text { Animal used } \\
\text { of the drugs }\end{array}$ & $\begin{array}{l}\text { Routes of } \\
\text { Administration }\end{array}$ & Local/Imported \\
\hline $\begin{array}{l}\text { Alenzol/K } \\
\text { Tablet }\end{array}$ & $\begin{array}{l}\text { Albendazole }(375 \\
\mathrm{mg})\end{array}$ & Sheep & Oral & Local \\
\hline $\begin{array}{l}\text { Anfenzan } \\
\text { Tablet }\end{array}$ & $\begin{array}{l}\text { Oxyclozanide }(600 \\
\text { mg) } \\
\text { Oxfendazole } \\
\text { mg })\end{array}$ & Sheep, Cattle & Oral & Local \\
\hline $\begin{array}{l}\text { Fortezol } \\
\text { Tablet }\end{array}$ & $\begin{array}{l}\text { Oxyclozanide }(900 \\
\text { mg) } \\
\text { Oxfendazole } \\
\text { mg })\end{array}$ & $\begin{array}{l}\text { Sheep, Lamb, } \\
\text { Cattle, Calf, } \\
\text { Beef, Heifers, } \\
\text { Moss }\end{array}$ & Oral & Local \\
\hline $\begin{array}{l}\text { Levadif } \\
\text { Granule for } \\
\text { Oral Solution }\end{array}$ & Levamisole (15 g) & $\begin{array}{l}\text { Owl, Goose, } \\
\text { Budgerigar, } \\
\text { Duck, Parrot, } \\
\text { Ostrich, } \\
\text { Chick }\end{array}$ & Oral & Local \\
\hline $\begin{array}{l}\text { Levatrizol } \\
\text { Tablet }\end{array}$ & $\begin{array}{l}\text { Levamisole } \\
(375 \mathrm{mg}) \\
\text { Triclabendazole } \\
(600 \mathrm{mg})\end{array}$ & $\begin{array}{l}\text { Calf, } \\
\text { Sheep, } \\
\text { Lamb, } \\
\text { Cattle }\end{array}$ & Oral & Local \\
\hline $\begin{array}{l}\text { Levazanid } \\
\text { Tablet }\end{array}$ & $\begin{array}{ll}\text { Levamisole } & (375 \\
\mathrm{mg}) & \\
\text { Oxyclozanide } & (750 \\
\mathrm{mg})\end{array}$ & $\begin{array}{l}\text { Calf, Beef, } \\
\text { Heifer, Moss, } \\
\text { Sheep, Cattle }\end{array}$ & Oral & Local \\
\hline $\begin{array}{l}\text { Mamdezol } \\
\text { Tablet }\end{array}$ & $\begin{array}{ll}\text { Oxyclozanite } & (750 \\
\mathrm{mg}) & \\
\text { Oxfendazole } & (375 \\
\mathrm{mg})\end{array}$ & $\begin{array}{l}\text { Calf, Veal, } \\
\text { Heifer, Moss, } \\
\text { Sheep, Lamb }\end{array}$ & Oral & Local \\
\hline $\begin{array}{l}\text { Nilverm } \\
\text { Powder for } \\
\text { Oral Solution }\end{array}$ & $\begin{array}{l}\text { Levamisole }(150 \\
\mathrm{mg})\end{array}$ & $\begin{array}{l}\text { Owl, Goose, } \\
\text { Budgerigar, } \\
\text { Duck, Parrot, } \\
\text { Ostrich, } \\
\text { Chick, } \\
\text { Turkey (fed } \\
\text { for meat), } \\
\text { Chicken (fed } \\
\text { for meat), } \\
\text { Pheasant }\end{array}$ & Oral & Local \\
\hline Nilzan & Levamisole & Sheep, Cattle & Oral & Local \\
\hline
\end{tabular}




\begin{tabular}{|c|c|c|c|c|}
\hline Tablet & $\begin{array}{l}\text { mg) } \\
\text { Oxyclozanite } \quad(300 \\
m g)\end{array}$ & & & \\
\hline $\begin{array}{l}\text { Okzan-F } \\
\text { Tablet }\end{array}$ & $\begin{array}{ll}\text { Oxyclozanite } & (750 \\
\text { mg) } & \\
\text { Oxfendazole } & (250 \\
\text { mg }) & \end{array}$ & Sheep, Cattle & Oral & Local \\
\hline $\begin{array}{l}\text { Profender } \\
\text { Dripping } \\
\text { Solution }\end{array}$ & $\begin{array}{ll}\text { Praziquantel } & (85.8 \\
\text { mg) } & \\
\text { Emodepsite } & (21.4 \\
\text { mg }) & \end{array}$ & Cat & $\begin{array}{l}\text { Topical, } \\
\text { Dermatologic }\end{array}$ & Imported \\
\hline $\begin{array}{l}\text { Tenyasid } \\
\text { Capsule }\end{array}$ & $\begin{array}{l}\text { Pyrantel embonate } \\
(144 \mathrm{mg}) \\
\text { Praziquantel } \\
\text { mg) Febantel }(150 \\
\text { mg) }\end{array}$ & Dog & Oral & Local \\
\hline $\begin{array}{l}\text { Vetalben } \\
\text { Tablet }\end{array}$ & $\begin{array}{l}\text { Albendazole }(200 \\
\mathrm{mg})\end{array}$ & Sheep, Cattle & Oral & Local \\
\hline
\end{tabular}

Table 7: MFAL Licensed Ectoparasiticides

\begin{tabular}{|l|l|l|l|l|}
\hline Drugs & Active substances & $\begin{array}{l}\text { Animal used } \\
\text { of the drugs }\end{array}$ & $\begin{array}{l}\text { Routes of } \\
\text { Administration }\end{array}$ & $\begin{array}{l}\text { Local/ } \\
\text { Imported }\end{array}$ \\
\hline $\begin{array}{l}\text { Bayticol \%1 } \\
\text { Solution for } \\
\text { pouring }\end{array}$ & Flumethrin $(10 \mathrm{mg})$ & Cattle & $\begin{array}{l}\text { Topical, } \\
\text { Dermatologic }\end{array}$ & Local \\
\hline $\begin{array}{l}\text { Flugon } \\
\text { Solution for } \\
\text { pouring }\end{array}$ & Flumethrin $(10 \mathrm{mg})$ & Sheep, Cattle & $\begin{array}{l}\text { Topical, } \\
\text { Dermatologic }\end{array}$ & Local \\
\hline
\end{tabular}

Table 8: MFAL Licensed Antiprotozoals

\begin{tabular}{|l|l|l|l|l|}
\hline Drugs & $\begin{array}{l}\text { Active } \\
\text { substances }\end{array}$ & $\begin{array}{l}\text { Animal used of } \\
\text { the drugs }\end{array}$ & $\begin{array}{l}\text { Routes of } \\
\text { Administration }\end{array}$ & Local/Imported \\
\hline $\begin{array}{l}\text { Fumidil-B } \\
\begin{array}{l}\text { Powder for } \\
\text { Syrup }\end{array}\end{array}$ & $\begin{array}{l}\text { Fumagillin } \\
(500 \mathrm{mg})\end{array}$ & Bee & Oral & Local \\
\hline $\begin{array}{l}\text { Coccil Oral } \\
\text { Suspension }\end{array}$ & $\begin{array}{l}\text { Toltrazuril (50 } \\
\mathrm{mg})\end{array}$ & Sheep & Oral & Imported \\
\hline
\end{tabular}

Table 9: MFAL Licensed Endectocides

\begin{tabular}{|l|l|l|l|l|}
\hline Drugs & $\begin{array}{l}\text { Active } \\
\text { substances }\end{array}$ & $\begin{array}{l}\text { Animal used of } \\
\text { the drugs }\end{array}$ & $\begin{array}{l}\text { Routes of } \\
\text { Administr } \\
\text { ation }\end{array}$ & $\begin{array}{l}\text { Local/ } \\
\text { Imported }\end{array}$ \\
\hline $\begin{array}{l}\text { Ultramec Forte \%2 } \\
\text { Injectable Solution }\end{array}$ & $\begin{array}{l}\text { Ivermectin (20 } \\
\mathrm{mg})\end{array}$ & Shhep, Cattle & IM, SC & Local \\
\hline $\begin{array}{l}\text { Eprecis Injectable } \\
\text { Solution }\end{array}$ & $\begin{array}{l}\text { Eprinomektin( } \\
10 \mathrm{mg})\end{array}$ & Cattle & $\mathrm{SC}$ & Local \\
\hline
\end{tabular}

Table 10: MFAL Licensed Antiinflammatory, Analgesics and Antirheumatic Drugs

\begin{tabular}{|l|l|l|l|l|}
\hline Drugs & $\begin{array}{l}\text { Active } \\
\text { substances }\end{array}$ & $\begin{array}{l}\text { Animal used of } \\
\text { the drugs }\end{array}$ & $\begin{array}{l}\text { Routes of } \\
\text { Administr } \\
\text { ation }\end{array}$ & Local/Imported \\
\hline $\begin{array}{l}\text { Vetakort 4 mg } \\
\text { Injectable Solution }\end{array}$ & $\begin{array}{l}\text { Dexamethason } \\
\text { e ( } 4 \mathrm{mg})\end{array}$ & $\begin{array}{l}\text { Horse, Cat, Dog, } \\
\text { Cattle, Thai }\end{array}$ & $\begin{array}{l}\text { IV, IM, } \\
\text { SC, IA }\end{array}$ & Local \\
\hline
\end{tabular}




\begin{tabular}{|l|l|l|l|l|}
\hline & & & $\begin{array}{l}\text { (Intraartic } \\
\text { ular) }\end{array}$ & \\
\hline $\begin{array}{l}\text { Difaljin Injectable } \\
\text { Solution }\end{array}$ & $\begin{array}{l}\text { Metamizole } \\
\text { Sodium (500 } \\
\text { mg) }\end{array}$ & $\begin{array}{l}\text { Heifer, Thai, } \\
\text { Moss, Horse, Dog }\end{array}$ & IV & Local \\
\hline $\begin{array}{l}\text { Flunifen Injectable } \\
\text { Solution }\end{array}$ & $\begin{array}{l}\text { Flunixin (50 } \\
\text { mg) }\end{array}$ & Horse, Cattle & IV, IM & Imported \\
\hline $\begin{array}{l}\text { Novo-cyan } \\
\text { Injectable Solution }\end{array}$ & $\begin{array}{l}\text { Metamizole } \\
\text { Sodium (5000 } \\
\text { mg) } \\
\text { Lidocaine } \\
\text { hydrochloride } \\
(1.5 \%)\end{array}$ & Horse, Dog, Thai & IM & Local \\
& $\begin{array}{l}\text { Vitamin } \\
B_{12}(4000 \mathrm{mg}) \\
\text { Vitamin } \\
\mathrm{B}_{1}(1000 \mathrm{mg}) \\
\text { Vitamin } \\
(100 \mathrm{mg})\end{array}$ & & & \\
& & & \\
\end{tabular}

Table 11: MFAL Licensed Gastrointestinal System Drugs

\begin{tabular}{|l|l|l|l|l|}
\hline Drugs & Active substances & $\begin{array}{l}\text { Animal of } \\
\text { used of } \\
\text { the drugs }\end{array}$ & $\begin{array}{l}\text { Routes } \\
\text { Administration }\end{array}$ & Local/Imported \\
\hline $\begin{array}{l}\text { Asivet } \\
\text { Emulsion }\end{array}$ & Simeticone $(30 \mathrm{mg})$ & $\begin{array}{l}\text { Horse, } \\
\text { Goat, } \\
\text { Sheep, } \\
\text { Cattle, } \\
\text { Thai }\end{array}$ & Oral & Local \\
\hline $\begin{array}{l}\text { Mete Oral } \\
\text { Emulsion }\end{array}$ & $\begin{array}{l}\text { Dimethylpolysiloxane } \\
(\% 2.5)\end{array}$ & $\begin{array}{l}\text { Horse, } \\
\text { Calf, Goat, } \\
\text { Sheep, } \\
\text { Thai }\end{array}$ & Oral & \\
& & & Local \\
\hline
\end{tabular}

Table 12: MFAL Licensed Anticholinergic Drugs

\begin{tabular}{|l|l|l|l|l|}
\hline Drugs & $\begin{array}{l}\text { Active } \\
\text { substances }\end{array}$ & $\begin{array}{l}\text { Animal used } \\
\text { of the drugs }\end{array}$ & $\begin{array}{l}\text { Routes of } \\
\text { Administration }\end{array}$ & Local / Imported \\
\hline $\begin{array}{l}\text { Vetaş Atropin } \\
\% 2 \text { Injectable }\end{array}$ & $\begin{array}{l}\text { Atropine } \\
\text { Sulfate }\end{array}$ & $\begin{array}{l}\text { Horse, Calf, IV, SC } \\
\text { Goat, Sheep, } \\
\text { Beef, Heifer, } \\
\text { Moss, } \\
\text { Capricorn }\end{array}$ & & Local \\
& & & \\
& & & \\
\hline
\end{tabular}

Table 13: MFAL Licensed Analeptic Drugs

\begin{tabular}{|l|l|l|l|l|}
\hline Drugs & $\begin{array}{l}\text { Active } \\
\text { substances }\end{array}$ & $\begin{array}{l}\text { Animal } \\
\text { used of the } \\
\text { drugs }\end{array}$ & $\begin{array}{l}\text { Routes } \\
\text { Administration }\end{array}$ & Local/ Imported \\
\hline $\begin{array}{l}\text { Kafedif } \\
\text { Injectable } \\
\text { Solution }\end{array}$ & $\begin{array}{l}\text { Caffeine } \\
\text { mg) } \\
\text { Sodium } \\
\text { Benzoate } \\
\text { mg) }\end{array}$ & $\begin{array}{l}\text { Horse, Goat, } \\
\text { Cat, Cattle, } \\
\text { Dog }\end{array}$ & & LC \\
\hline
\end{tabular}


Table 14: MFAL Licensed Genito Urinary System Drugs and Sex Hormones

\begin{tabular}{|l|l|l|l|l|}
\hline Drugs & $\begin{array}{l}\text { Active } \\
\text { substances }\end{array}$ & $\begin{array}{l}\text { Animal of } \\
\text { used of } \\
\text { the drugs }\end{array}$ & $\begin{array}{l}\text { Routes } \\
\text { Administration }\end{array}$ & Local/Imported \\
\hline $\begin{array}{l}\text { Enzaprost-T } \\
\text { Injectable } \\
\text { Solution }\end{array}$ & $\begin{array}{l}\text { Dinoprost } \\
\mathrm{mg})\end{array}$ & $\begin{array}{l}\text { Horse, } \\
\text { Cattle }\end{array}$ & IM & Imported \\
\hline $\begin{array}{l}\text { Prid-delta } \\
\text { Intravajinal }\end{array}$ & $\begin{array}{l}\text { Progesterone } \\
(1.55 \mathrm{~g})\end{array}$ & Cattle & Intravaginal & Imported \\
\hline
\end{tabular}

Table 15: MFAL Licensed Hormones

\begin{tabular}{|l|l|l|l|l|}
\hline Drugs & $\begin{array}{l}\text { Active } \\
\text { substances }\end{array}$ & $\begin{array}{l}\text { Animal of } \\
\text { used of } \\
\text { the drugs }\end{array}$ & $\begin{array}{l}\text { Routes } \\
\text { Administration }\end{array}$ & Local/Imported \\
\hline $\begin{array}{l}\text { Ovarelin } \\
\text { Injectable } \\
\text { Solution }\end{array}$ & $\begin{array}{l}\text { Gonadorelin } \\
\text { diacetate } \\
\text { tetrahydrate } \\
\text { mcg) }\end{array}$ & Cattle & IV, IM & Imported \\
\hline $\begin{array}{l}\text { Regulin } \\
\text { Implant }\end{array}$ & $\begin{array}{l}\text { Melatonin } \\
\text { mg) }\end{array}$ & Sheep & SC & Imported \\
\hline
\end{tabular}

Table 16: MFAL Licensed Non-Therapeutic Products

\begin{tabular}{|c|c|c|c|c|}
\hline Drugs & $\begin{array}{l}\text { Active } \\
\text { substances }\end{array}$ & $\begin{array}{l}\text { Animal } \\
\text { used of the } \\
\text { drugs }\end{array}$ & $\begin{array}{l}\text { Routes of } \\
\text { Administration }\end{array}$ & Local/Imported \\
\hline $\begin{array}{l}\text { Cevamune } \\
\text { Effervescent } \\
\text { tablet }\end{array}$ & $\begin{array}{l}\text { Patent Blue V } \\
(1200 \mathrm{mg}) \\
\text { Sodium } \\
\text { Thiosulfate } \\
(1500 \mathrm{mg})\end{array}$ & $\begin{array}{l}\text { Owl, Chick, } \\
\text { Ostrich, } \\
\text { Dogan, } \\
\text { Pigeon, } \\
\text { Canary, } \\
\text { Pheasant, } \\
\text { Love Bird, } \\
\text { Duck, } \\
\text { Parrot, } \\
\text { Hawk, } \\
\text { Goose, } \\
\text { Chicken }\end{array}$ & Oral & Imported \\
\hline
\end{tabular}

Table 17: Ministry of Health Licensed Antiseptics and Disinfectants

\begin{tabular}{|c|c|c|c|c|}
\hline Drugs & $\begin{array}{l}\text { Active } \\
\text { substances }\end{array}$ & $\begin{array}{l}\text { Microorganisms } \\
\text { that drugs is } \\
\text { effective }\end{array}$ & $\begin{array}{l}\text { Routes of } \\
\text { Administration }\end{array}$ & Local/Imported \\
\hline $\begin{array}{l}\text { Dezen \%20 } \\
\text { Solution }\end{array}$ & $\begin{array}{l}\text { Benzalkonium } \\
\text { Chloride }(20 \%)\end{array}$ & $\begin{array}{l}\text { E.coli, S.aureus, } \\
\text { P.aeruginosae, } \\
\text { C.albicans, } \\
\text { S.pyogenes }\end{array}$ & $\begin{array}{l}\text { Surface (Sliding, } \\
\text { Spraying, Dipping) }\end{array}$ & Local \\
\hline
\end{tabular}

(RxMediapharma 2017) Percentage distributions of the preparations according to pharmacological groups are given in Figure 1.

In this study, Ministry of Health Licensed 1; The Ministry of Food, Agriculture and Livestock has registered 79 veterinary drugs, 56 of which are local and 23 of which are imported. The Ministry of Food, Agriculture and Livestock Licensed and Ministry of Health lists 80 licensed preparations and describes the active 
substances, animals used, drug form, route of application, local or imported status contained in the preparations. There are also 364 Ministry of Food, Agriculture and Livestock Marketing Permits and 298 Ministry of Agriculture and Rural Affairs licensed drugs that are not included in the scope of the study. Ministry of Food, Agriculture and Livestock Ministry of Health, when the licensed drugs are grouped according to their pharmacological effects, the licensed drug group which has the highest number with $43.75 \%$ is antibiotics and this percentage is shown by the percentages that anthelmintics followed by $16.25 \%$. Vitamins with a ratio of $10 \%$; Antiinflammatory Analgesic Antirheumatic Drugs in a ratio of 5\%; Mineral Supports, Electrolytes, Ectoparasites, Gastrointestinal System Drugs, Hormones, Genitourinary System Drugs and Sex Hormones; Anticholinergic Drugs, Analeptic Drugs, Antiseptic and Disinfectants and Non-Therapeutic Products were observed in $1.25 \%$ (Figure 1).
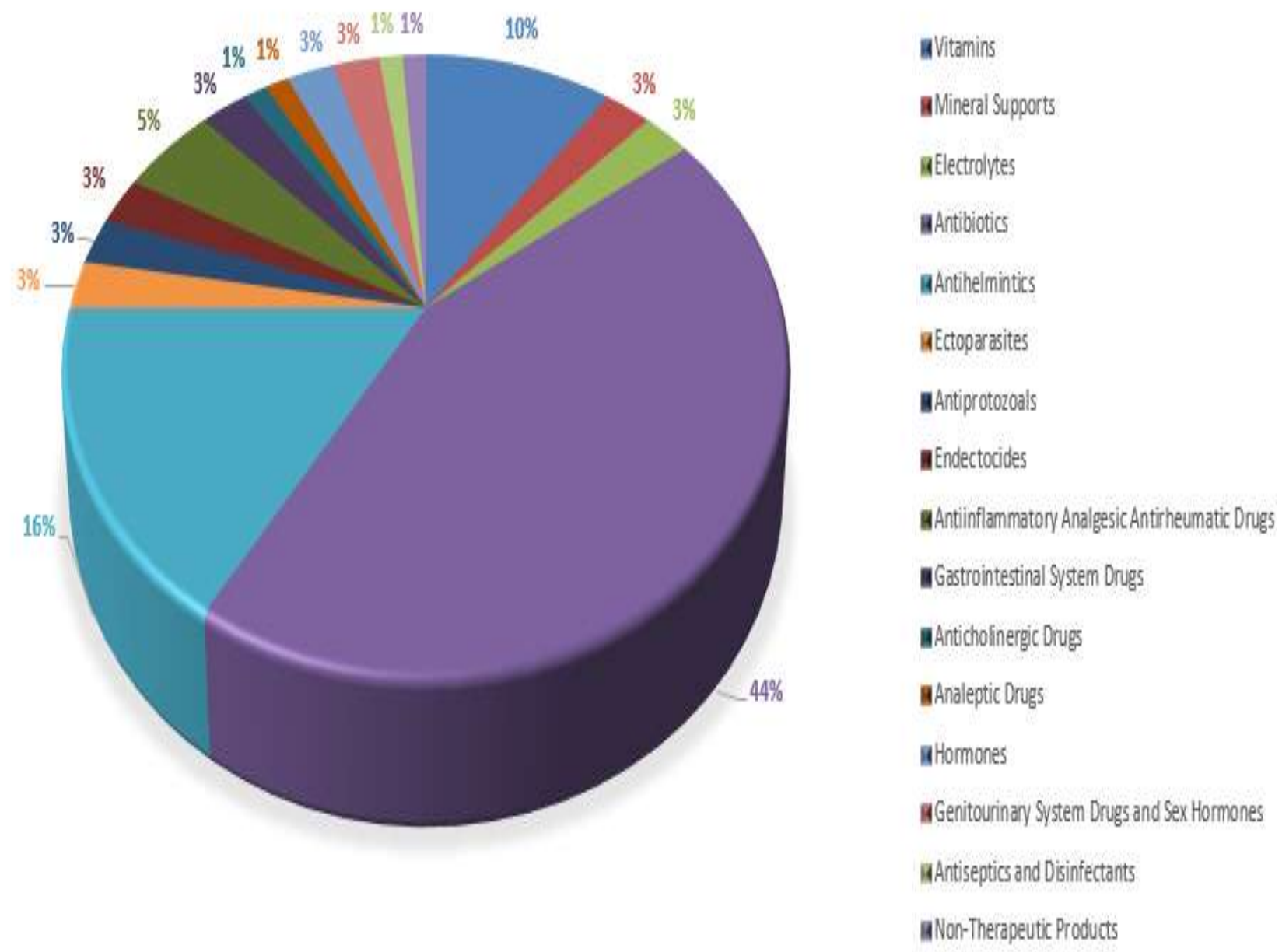

Figure 1: Distribution chart of the Ministry of Food, Agriculture and Livestock licensed drugs of the according to pharmacological groups

\section{Discussion and Conclusion}

In veterinary medicine; the use of medicines in order to serve different purposes, especially in the treatment of animal diseases (Yarsan 2015). When the history of veterinary drug use in the world is examined, it is not known how and how veterinary drugs are used for the first time, but it is accepted that they have been used since the localation of animals (Ozturk 2002). After the localation of animals, it has been seen in various books where veterinary drug recipes have been developed and used since ancient times. One of these books, İbn Baytar, was mentioned together with veterinary and human envi- ronment drugs in the "Kitab al-Cami fi-l-Adviya al-Mufrada" (Asil 2001, Baytop 1985, Erk 1955, Sarton 1950). It is noteworthy that in some of the works called "Baytarname" the information about the veterinary medicine belonging to the Islamic Civilization Age is reached and the information about how and how the medicines can be used in some ways is presented with pictorial descriptions (Dincer and Erk 1967, Dincer 1982). Considering the history of veterinary drug use in Turkey in 1842 opened the first Veterinarians have begun to use drugs with the school, although the sectoral sense of progress after 1950 as başlamıştır.1950'l year has also increased the veterinary drug production with the increase of local pharmaceutical factories (Ozturk 2002). 
Production and licensing of veterinary medicines in our country is carried out by the Ministry of Food, Agriculture and Livestock and the Ministry of Health. The production and use of unlicensed veterinary medicines from the Ministry is legally prohibited. The license of the licensed drug is canceled when it is considered inadequate in terms of efficiency and safety. The availability of licensed veterinary medicines, pharmacy and clinic sales areas may be possible with quality, reliability and efficacy criteria (3). Ministry of Health Licensed 1 in our work; The Ministry of Food, Agriculture and Livestock has registered 79 veterinary drugs, 56 of which are local and 23 of which are imported. There are also 364 Ministry of Food, Agriculture and Livestock Marketing Permits and 298 Ministry of Agriculture and Rural Affairs licensed drugs that are not included in the scope of the study.

In addition, the use of veterinary medicines on the market in veterinarian control and in the counseling of pharmacists who have been trained in this field is extremely important in terms of community health. Unconscious use of these drugs can have serious consequences. For this reason, it is necessary for the veterinarians to record the disease situation in animals, the effect of the applied drugs, the dosage and the frequency of application, the information about the target animal and the indication status in electronic form. This study used in pharmacies in Turkey rxmediapharma ${ }^{\circledR}$ maintained under active electronically scanned and recorded using a 2017 veterinary drugs have been listed. According to this result, the most licensed drug group is antibiotics and this is followed by anthelmintics, vitamins, antiinflamatuar analgesic antirheumatic drugs, mineral supplements, electrolytes, ectoparasitists, gastrointestinal system drugs, hormones, genitourinary system drugs and sex hormones, anticholinergic drugs, antiseptic and disinfectants and nontherapeutic products have been identified.

In the framework of the results obtained from this research in our country in the field of animal health, animal diseases and drug database that is used to perform an effective fight against pests (rxmediapharma ${ }^{\circledR}$ ) organized by reference, veterinary medicines that are available in pharmacies in Turkey and their active substances, animals used drugs form, method of administration, local or imported situation is considered as the first work in the collective, given that veterinarians and pharmacists are expected to provide great benefits for their work. This research is thought to be a source of hope in protecting society and animal health, together with future work in this regard.

\section{Acknowledgment}

Erciyes University, Faculty of Veterinary Medicine, Department of Pharmacology and Toxicology, Professor. Dr. We thank Murat KANBUR.

\section{References}

[1] Asil E (2001) Dünden bugüne eczacıl1k. MISED Dergisi. 4-19.

[2] Baytop T (1985) Türk eczacılık tarihi. İstanbul: İstanbul Üniversitesi Yayınları.

[3] Dincer F (1982) Türk folklorunda veteriner hekimliğin yazılı kaynakları. II. Milletlerarası Türk Folklor Kongresi Bildirileri, Başbakanlık Basımevi 4: 123-130.

[4] Dincer F, Erk N (1967) XV inci ya da XVI nc1 yüzyıla ait olduğu sanılan bir baytarname incelemesi. Ankara University Veterinary Faculty Journal 14(2): 117-139.

[5] DPT (2001) Sekizinci bes yıllık kalkınma planı ilaç sanayi özel ihtisas komisyonu raporu. Yayın No:2540- ÖİK: 556.

[6] Erk N (1955) XVIncı asır veteriner hekimliği ve bu asra ait eserler. Ankara: Yeni Desen Matbaas1.

[7] Öztürk R (2002) Türkiye'de veteriner ilaç sanayiinin tarihsel gelişimi. PhD’s Thesis, Turkey: Ankara Üniversitesi

[8] Sarton G (1950) Introdiction to history of science. Washington: The Williams AND Wilkins Company.

[9] Yarsan E (2015) Kedi ve köpek hekimliği. Ankara: Günes Tıp Kitapevleri 